\section{SOI: $\underline{1.1 / \mathrm{TAS}}$ DOI: $\underline{10.15863 / \mathrm{TAS}}$ International Scientific Journal Theoretical \& Applied Science}

\author{
p-ISSN: 2308-4944 (print) e-ISSN: 2409-0085 (online) \\ Year: $2015 \quad$ Issue: 03 Volume: 23 \\ Published: $30.03 .2015 \quad$ http://T-Science.org
}

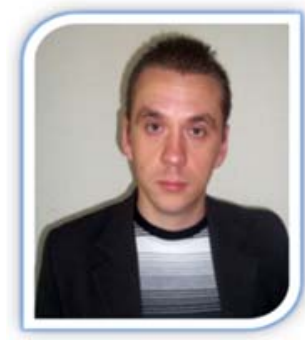

Denis Alexandrovich Chemezov Master of Engineering and Technology, Corresponding member of International

Academy TAS, Lecturer of Vladimir Industrial College, Russia chemezov-da@yandex.ru

SECTION 7. Mechanics and machine construction.

\title{
THE CHOICE OF THE OPTIMAL ROUND/RING CROSS-SECTION STEEL RODS RUNNING TORSION
}

Abstract: The article presents a research of the values of strain and stress of the steel rods of round and ring cross-sections in the application to them of torsion torque. Defined the optimal cross-section, providing the greatest strength of the rod and rational material savings.

Key words: test sample, strain, stress, displacement.

Language: Russian

Citation: Chemezov DA (2015) THE CHOICE OF THE OPTIMAL ROUND/RING CROSS-SECTION STEEL RODS RUNNING TORSION. ISJ Theoretical \& Applied Science 03 (23): 44-48.

Soi: http://s-o-i.org/1.1/TAS*03(23)9 Doi: crossef http://dx.doi.org/10.15863/TAS.2015.03.23.9

УДК 620.162.3

\section{ВЫБОР ОПТИМАЛЬНОГО КРУГЛОГО/КОЛЬЦЕВОГО ПОПЕРЕЧНОГО СЕЧЕНИЯ СТАЛЬНЫХ СТЕРЖНЕЙ РАБОТАЮЩИХ НА КРУЧЕНИЕ}

Аннотация: В статье представлено исследование величин деформирования и напряжения стальных стержней круглого и кольцевого поперечных сечений при приложении на них вращающего момента. Определено оптимальное поперечное сечение, обеспечивающее наибольшую прочность стержня и рациональную экономию материала.

Ключевые слова: образеи, деформация, напряжение, перемещение.

Прочностные характеристики деталей машин определяются путем испытаний образцов на изгиб, растяжение, сжатие, кручение и др. Испытания на кручение цилиндрических образцов, изготовленных из конструкционной стали, выполняются для нахождения модуля сдвига, предела пропорциональности, предела текучести, условного и истинного предела прочности, максимального остаточного сдвига и характера разрушения материала [1]. Величины деформации и напряжения материала будут зависеть от ряда факторов, одним из которых является геометрическая форма детали в поперечном сечении. Детали, работающие на кручение, могут быть сплошными (круглого поперечного сечения) и полые (кольцевого поперечного сечения) для облегчения изделия и соответственно экономии материала. Известно, что по прочности детали с кольцевым поперечным сечением не уступают деталям с круговым поперечным сечением. Расчет напряженно-деформированного состояния материала и последующий анализ результатов, позволит выбрать оптимальное круглое/ кольцевое поперечное сечение стальных стержней.

Испытаниям на кручение подвергались пять цилиндрических образцов наружными диаметрами 50 мм, которые одним свободным концом были заделаны в стену. Первый испытуемый образец имел круглое поперечное сечение, остальные - кольцевые поперечные сечения с различными внутренними диаметрами. Образцы изготавливались из одного материала конструкционной стали Ст3сп (DIN S235J2G3) имеющей следующие физико-механические

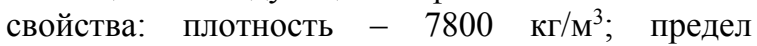
текучести $-2,35 \times 10^{8} \mathrm{H} / \mathrm{M}^{2}$; предел прочности при растяжении - $3,6 \times 10^{8} \mathrm{H} / \mathrm{M}^{2}$; модуль упругости $-2,1 \times 10^{11} \mathrm{H} / \mathrm{M}^{2}$; коэффициент 
Пуассона - 0,28; модуль сдвига - 7,9 $\times 10^{10} \mathrm{H} / \mathrm{M}^{2}$; коэффициент теплового расширения $-1,1 \times 10^{-5}$
$\mathrm{K}^{-1}$. Объемные свойства образцов представлены в табл. 1.

Объемные свойства образцов.

\begin{tabular}{|c|c|c|}
\hline № образца & Масса, $\mathbf{\kappa} \boldsymbol{\Gamma}$ & Объем, $\mathbf{~}^{\mathbf{3}}$ \\
\hline 1 & 7,657 & 0,00098 \\
\hline 2 & 7,351 & 0,00094 \\
\hline 3 & 6,432 & 0,00082 \\
\hline 4 & 4,9 & 0,00062 \\
\hline 5 & 2,756 & 0,00035 \\
\hline
\end{tabular}

Таблица 1

К образцам был приложен вращающий момент величиной $250 \mathrm{H} \times$ м. Графическое изображение постановки исследования представлено на рис. 1.

Испытания осуществлялись способом имитационного нагружения твердотельных объемных моделей образцов в компьютерной программе SolidWorks. Решение выполнялось адаптивным методом ( $h$ - метод) с целевой точностью $98 \%$.

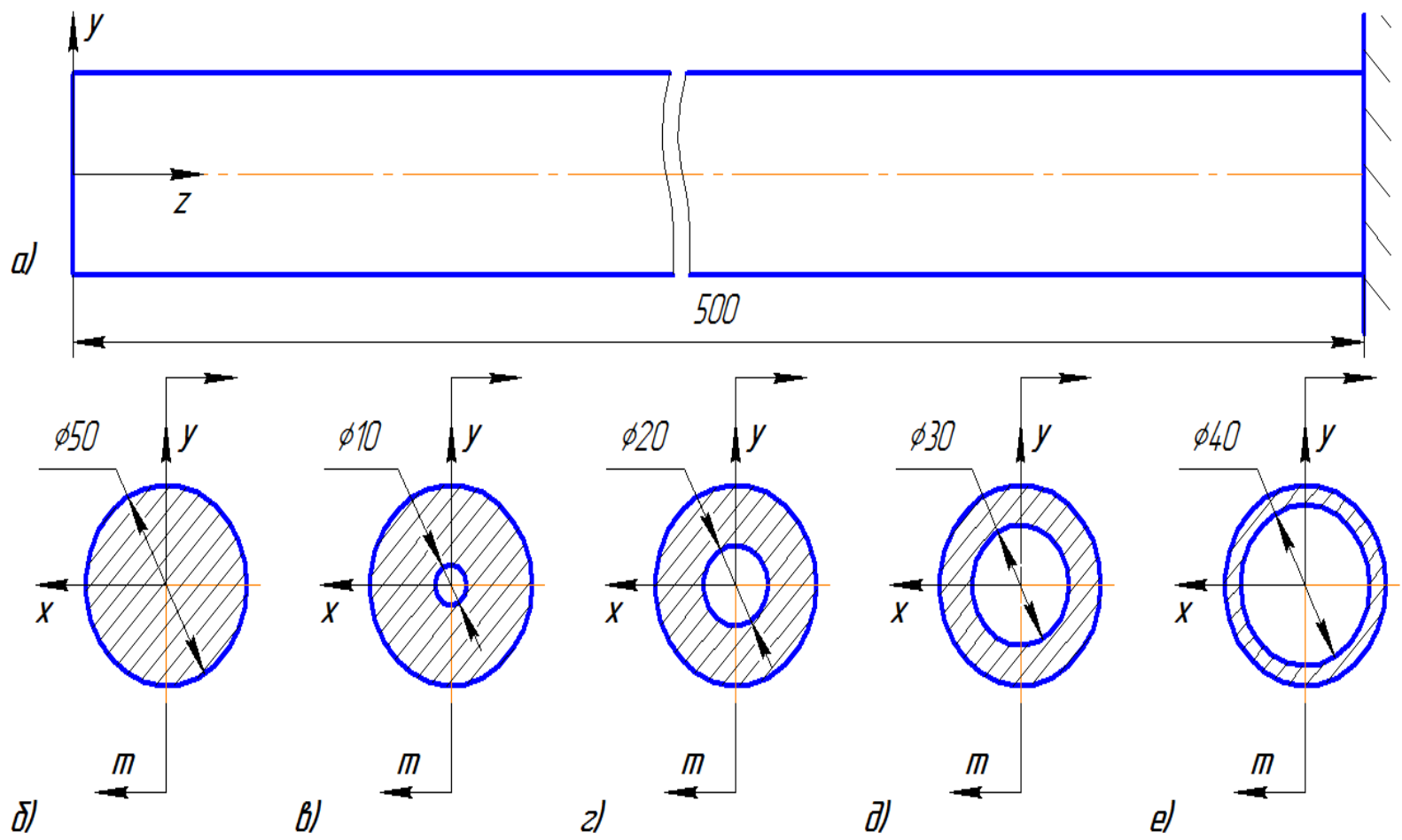

Рисунок 1 - Постановка исследования: а - главный вид круглого стержня имеющего жесткую заделку с одного конца; б - геометрическая форма и размер образца 1 в поперечном сечении; в геометрическая форма и размер образца 2 в поперечном сечении; г - геометрическая форма и размер образца 3 в поперечном сечении; д - геометрическая форма и размер образца 4 в поперечном сечении; е - геометрческая форма и размер образца 5 в поперечном сечении. $m$ - наружный вращающий момент равный $250 \mathrm{H} \times$ м.

Результаты расчета представлены в сводной табл. 2: образцов
1) напряженное состояние материала a) напряжение Von Mises $(V O N)$ - величина напряжения, рассчитанная исходя из составляющих напряжения твердого тела [2];

б) нормальные напряжения по координатным осям $(S X, S Y, S Z)$ - составляющие 
(компоненты) напряженного состояния твердого тела в рассматриваемой точке [3];

в) сдвиги по координатным осям на плоскостях (TXY, $T X Z, T Y Z)$ - составляющие (компоненты) напряженного состояния тела, определяющие совокупность напряжений, возникающих по различным осям и в различных плоскостях;

г) главные напряжения $(P 1, P 2, P 3)-3$ компонента нормальных напряжений определяемые в направлениях без учета касательных напряжений;

д) интенсивность напряжения (INT) величина, определяющая касательное напряжение на элементарной площадке, одинаково наклонённой к главным осям напряжений в точке [4];

е) ошибка энергетической нормы $(E R R)-$ степень точности нормы энергии напряженного материала.

2) перемещение образцов относительно начального положения

a) перемещения по координатным осям ( $U X$, $U Y, \quad U Z)$ - составляющие результирующего перемещения;

б) результирующее перемещение (URES) векторная сумма последовательных перемещений образцов по координатным осям относительно начального положения;

в) силы реакций по координатным осям $(R F X, \quad R F Y, \quad R F Z)$ - силы, направленные перпендикулярно поверхности соприкосновения [5];

г) результирующая сила реакции (RFRES) алгебраическая сумма сил реакций по координатным осям;

3) деформированное состояние материала образцов

a) нормальные деформации по координатным осям (EPSX, EPSY, EPSZ) составляющие эквивалентной деформации материала. Определяются как приращение длины твёрдого тела к исходной длине в рассматриваемом направлении;

б) эквивалентная деформация (ESTRN) алгебраическая сумма нормальных деформаций и сдвигов по координатным осям;

в) сдвиги по координатным осям на плоскостях $(G M X Y, \quad G M X Z, \quad G M Y Z) \quad-$ составляющие (компоненты) деформированного состояния тела, определяющие совокупность деформаций, возникающих по различным осям и в различных плоскостях;

г) плотность энергии деформации (SEDENS) - количество энергии на единицу объёма твердого тела [6];

д) суммарная энергия деформации $(E N E R G Y)$ - суммарная работа, приходящаяся на единицу объема твердого тела [7];

е) деформации в главных направлениях (E1, $E 2, E 3)$ - деформации твердого тела в трех ортогональных направлениях (главные оси деформации - максимум, минимум и минимакс);

4) нагруженное состояние испытуемых образцов

a) design insight - процентное выражение нагруженных участков образцов от общего объема стержней.

5) запас прочности материала образцов

a) по критерию максимального напряжения von Mises $(F O S 1,2)$ - основывается на теории Мизес-Хенки. Пластичный материал начинает повреждаться в местах, где напряжение по Мизесу становится равным предельному напряжению. В большинстве случаев, предел текучести используется в качестве предельного напряжения [8];

б) по критерию максимального напряжения при сдвиге (Tresca) (FOS3,4) - прогнозирует разрушение материала в случаях, когда абсолютный максимум напряжения сдвига достигнет нагрузки, которая вызывает повреждение материала [9].

Таблица 2

Максимальные значения напряжения, перемещения относительно начального положения и деформации материала образцов под нагрузкой.

\begin{tabular}{|c|c|c|c|c|c|}
\hline $\begin{array}{c}\text { Параметр, } \\
\text { единица измерения }\end{array}$ & 1 образец & 2 образец & 3 образец & 4 образец & 5 образец \\
\hline Напряжение von Mises, $\mathrm{H} /$ мм $^{2}$ & 18,064 & 18,068 & 18,509 & 20,638 & 30,19 \\
\hline Нормальное напряжение X, Н/мм² & 0,146 & 0,166 & 0,141 & 0,144 & 0,148 \\
\hline Нормальное напряжение Y, Н/мм² & 0,154 & 0,158 & 0,143 & 0,143 & 0,151 \\
\hline Нормальное напряжение Z, H/мм² & 0,031 & 0,061 & 0,023 & 0,018 & 0,036 \\
\hline Сдвиг по Y на плоскости YZ, H/Mм² & 0,137 & 0,131 & 0,135 & 0,133 & 0,134 \\
\hline Сдвиг по Z на плоскости $\mathrm{YZ}, \mathrm{H} / \mathrm{Mм}^{2}$ & 10,35 & 10,383 & 10,666 & 11,866 & 17,386 \\
\hline Сдвиг по Z на плоскости $\mathrm{XZ}, \mathrm{H} / \mathrm{Mм}^{2}$ & 10,407 & 10,424 & 10,643 & 11,865 & 17,373 \\
\hline 1-ое главное напряжение, Н/мм² & 10,43 & 10,434 & 10,691 & 11,91 & 17,43 \\
\hline 2-ое главное напряжение, $\mathrm{H} / \mathrm{MM}^{2}$ & 0,058 & 0,061 & 0,022 & 0,016 & 0,034 \\
\hline
\end{tabular}

ISPC The Best of European Innovations, 


\begin{tabular}{|c|c|c|c|c|c|}
\hline 3-ее главное напряжение, Н/мм² & $-0,0002$ & $9,85 \times 10^{-5}$ & $-0,0001$ & $-0,0001$ & 0,00075 \\
\hline Интенсивность напряжения, Н/мм² & 20,86 & 20,863 & 21,373 & 23,831 & 34,86 \\
\hline Ошибка энергетической нормы & 0,575 & 0,736 & 0,67 & 0,553 & 0,323 \\
\hline Перемещение X, мм & 0,031 & 0,031 & 0,031 & 0,035 & 0,052 \\
\hline Перемещение Y, мм & 0,031 & 0,031 & 0,031 & 0,035 & 0,052 \\
\hline Перемещение Z, мм & $1,26 \times 10^{-6}$ & $9,85 \times 10^{-7}$ & $1,28 \times 10^{-6}$ & $1,09 \times 10^{-6}$ & $1,47 \times 10^{-6}$ \\
\hline Результирующее перемещение, мм & 0,031 & 0,031 & 0,031 & 0,035 & 0,052 \\
\hline Сила реакции $\mathrm{X}, \mathrm{H}$ & 34,548 & 31,407 & 30,167 & 33,066 & 48,963 \\
\hline Сила реакции Y, H & 34,632 & 33,53 & 34,33 & 34,648 & 42,409 \\
\hline Сила реакции $\mathrm{Z}, \mathrm{H}$ & 0,015 & 0,038 & 0,022 & 0,032 & 0,03 \\
\hline Результирующая сила реакции, Н & 37,706 & 34,062 & 37,464 & 37,385 & 54,149 \\
\hline Нормальная деформация по оси X & $8,32 \times 10^{-7}$ & $8,29 \times 10^{-7}$ & $8,20 \times 10^{-7}$ & $8,28 \times 10^{-7}$ & $8,49 \times 10^{-7}$ \\
\hline Нормальная деформация по оси Y & $8,26 \times 10^{-7}$ & $8,27 \times 10^{-7}$ & $8,29 \times 10^{-7}$ & $8,23 \times 10^{-7}$ & $8,45 \times 10^{-7}$ \\
\hline Нормальная деформация по оси Z & $7,26 \times 10^{-8}$ & $6,65 \times 10^{-8}$ & $7,52 \times 10^{-8}$ & $5,12 \times 10^{-8}$ & $6,73 \times 10^{-8}$ \\
\hline Эквивалентная деформация & $7,16 \times 10^{-5}$ & $7,17 \times 10^{-5}$ & $7,34 \times 10^{-5}$ & $8,20 \times 10^{-5}$ & 0,00012 \\
\hline Сдвиг по Y на плоскости YZ & $1,67 \times 10^{-6}$ & $1,6 \times 10^{-6}$ & $1,64 \times 10^{-6}$ & $1,6 \times 10^{-6}$ & $1,63 \times 10^{-6}$ \\
\hline Сдвиг по Z на плоскости YZ & 0,0001 & 0,00012 & 0,00013 & 0,00014 & 0,00021 \\
\hline Сдвиг по Z на плоскости XZ & 0,0001 & 0,00012 & 0,00013 & 0,00014 & 0,00021 \\
\hline $\begin{array}{c}\text { Плотность энергии деформации, } \\
(\mathrm{H} \times \mathrm{M}) / \mathrm{M}^{3} \\
\end{array}$ & 631,654 & 634,306 & 665,013 & 829,468 & 1780,14 \\
\hline $\begin{array}{c}\text { Суммарная энергия деформации, } \\
\text { H × м }\end{array}$ & $3,89 \times 10^{-6}$ & $4,64 \times 10^{-6}$ & $3,86 \times 10^{-6}$ & $5,69 \times 10^{-6}$ & $9,44 \times 10^{-6}$ \\
\hline $\begin{array}{c}\text { Деформации в 1, 2, } 3 \text { главных } \\
\text { направлениях }\end{array}$ & 0,00011 & 0,00012 & 0,00012 & 0,00013 & 0,0002 \\
\hline Design insight, $\%$ & 47,75 & 47,79 & 49,61 & 48,4 & 48,04 \\
\hline \multicolumn{6}{|c|}{ Запас прочности } \\
\hline $\begin{array}{l}\text { Максимальное напряжение von } \\
\text { Mises - предел текучести, H/мм² }\end{array}$ & 681790 & 316965 & 296822 & 410722 & 184565 \\
\hline $\begin{array}{c}\text { Максимальное напряжение von } \\
\text { Mises - предел прочности, H/Mм² }\end{array}$ & 1044444 & 485563 & 454707 & 629191 & 282738 \\
\hline 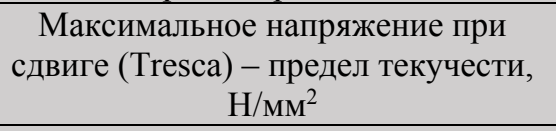 & 609410 & 275009 & 271164 & 356078 & 162314 \\
\hline $\begin{array}{c}\text { Максимальное напряжение при } \\
\text { сдвиге (Tresca) - предел прочности, } \\
\text { H/Mм² }\end{array}$ & 933564 & 421291 & 415400 & 545481 & 248651 \\
\hline
\end{tabular}

Проведена статистическая обработка результатов моделирования. Наименьшие значения напряжения, перемещения и деформации материала и наибольшие величины запаса прочности образцов принимались за один балл. Наибольшие значения параметров не учитывались.

1 образец - 22 балла (VON, TXZ,TYZ, P1, P3, INT, UX, UY, URES, RFZ, ESTRN, GMXZ, GMYZ, SEDENS, E1, E2, E3, Design insight, FOS1, FOS2, FOS3, FOS4).

2 образец - 17 баллов (VON, TXY, TXZ, TYZ, P1, INT, UX, UY, UZ, URES, RFY, RFRES, EPSY, ESTRN, GMXY, SEDENS, Design insight).

3 образец - 13 баллов (VON, SX, SY, TXZ, TYZ, P1, UX, UY, URES, RFX, EPSX, SEDENS, ENERGY). GMXY).

4 образец - 5 баллов (SY, SZ, P2, EPSZ,

5 образец - 1 балл $(E R R)$.

Принимая напряженно-деформированное состояние материала стержня круглого сечения за наименьшее, определено, что практически аналогичным характеристикам соответствуют второй и третий образцы, а по нескольким параметрам превосходят первый образец. Таким образом, оптимальное поперечное сечение стержня (1), работающего на кручение, должно выбираться в пределах (первое значение - для кольцевого поперечного сечения):

$$
(D \leq 0,4 d) ; d,
$$

где $d$ - наружный диаметр образца круглого поперечного сечения, мм; $D-$ внутренний диаметр образца, мм. 


\section{References:}

1. GOST 3565-80. Metally. Metod ispytaniya na kruchenie.

2. (2015) Konechno-elementny analiz v SolidWorks Simulation. Available: http://cadregion.ru/solidworkssimulation/konechno-elementnyj-analiz-vsolidworks-simulation.html (Accessed: 14.03.2015).

3. (2015) Napryajennoe i deformirovannoe sostoyanie chasticy tela. Available: http://www.soprotmat.ru/tns.htm (Accessed: 14.03.2015).

4. (2015) Intensivnost napryajeniy. Available: http://femto.com.ua/articles/part 1/1381.html (Accessed: 14.03.2015).

5. (2015) Sila normalnoy reakcii. Available: https://ru.wikipedia.org/wiki/Сила нормально й реакции (Accessed: 14.03.2015).

6. (2015) Plotnost energii. Available: https://ru.wikipedia.org/wiki/\% $\% \mathrm{CF} \% \mathrm{~EB} \% \mathrm{EE} \% \mathrm{~F}$
2\%ED $\%$ EE $\%$ F1\%F2\%FC $\%$ FD $\%$ ED $\%$ E5\%F0 $\% \mathrm{E} 3 \% \mathrm{E} 8 \% \mathrm{E} 8$ (Accessed: 14.03.2015).

7. (2015) Energiya deformacii. Available: http://lib.sernam.ru/book tupr.php?id $=92$ (Accessed: 14.03.2015).

8. (2015) Kriteriy: maksimalnoe napryajenie von Mises. Available: http://help.solidworks.com/2010/russian/Solid Works/cworks/LegacyHelp/Simulation/Checkin g_stresses/prot_vonm.html?id=979f07d4a8784 7c596e135c4b554344c\#Pg0 (Accessed: 14.03.2015).

9. (2015) Kriteriy: maksimalnoe napryajenie sdviga. Available: http://help.solidworks.com/2010/russian/Solid Works/cworks/LegacyHelp/Simulation/Checkin g stresses/prot shea.html 14.03.2015).
(Accessed: 